\title{
Chemical Exchange and Raman Line Broadening III. A Comment to [1]
}

\section{H. Strehlow}

\author{
Max-Planck-Institut für Biophysikalische Chemie, D-3400 Göttingen, Federal Republic of Germany
}

\section{Kinetics / Spectroscopy, Raman}

The question of whether the integrated intensities of vibrational spectra are changed due to fast chemical reactions [2] or whether they are not $[1]$ is discussed theoretically and experimentally.

\section{Theory}

An earlier treatment of line broadening in vibrational bands due to chemical exchange [2] was based on a second order perturbation theory. It differs already for the case of non-exchanging systems from the density matrix procedure given by $[1,3]$, although in this case the finite results are identical.

To clear the issue it may prove helpful to derive the relevant equations using a pure classical method [4].

We consider a linear harmonic oscillator as a simple model for a vibrating molecule. Without a damping force the oscillator will vibrate monochromatically for an infinite time. The equation of motion including a damping term reads

$m \frac{\mathrm{d}^{2} x}{\mathrm{~d} t^{2}}=-\omega_{0}^{2} \cdot x-2 \beta \frac{\mathrm{d} x}{\mathrm{~d} t}$.

The solution of Eq. (1) is

$x=x_{0} \exp (-\beta t) \exp \left(-\mathrm{i} \omega_{0} t\right)$

The averaged energy of the oscillator is

$W=m\left(\overline{\dot{x}^{2}}+\omega_{0}^{2} \cdot \overline{x^{2}}\right) / 2=W_{0} \exp (-2 \beta t)$.

Thus the energy decreases with a time-constant $1 / 2 \beta$ which is the lifetime of the oscillator.

The electric field $\boldsymbol{E}$ associated with the vibrating oscillator dipole is proportional to the amplitude of vibration and decays with a rate constant $\beta$. Therefore, with $t>0$

$$
E(t)=E_{0} \exp (-\beta t) \exp \left(-\mathrm{i} \omega_{0} t\right) .
$$

With a finite $\beta E$ is no longer monochromatic but extends over a frequency range of the order of $\beta$. This is realized by Fourier transforming Eq. (4) into the frequency domain

$\boldsymbol{E}(\omega)=\frac{E_{0} / 2 \pi}{\mathrm{i}\left(\omega_{0}-\omega\right)+\beta}$

The intensity distribution then becomes ([5]; compare [6])

$I(\omega)=$ const $\cdot E E^{*}=\frac{I_{0} \beta / \pi}{\left(\omega_{0}-\omega\right)^{2}+\beta^{2}}$.

The factor $I_{0}$ is equal to the total intensity $\int I(\omega) \mathrm{d} \omega$. Eq. (6) is a Lorentzian with half line width $2 \beta$ i.e. $\beta$ is the equivalent of $1 / T_{2}$ in the Bloch equations. Eq. (6) refers to the stationary spectrum of many stochastically excited oscillators. Abbreviating

$\alpha=\mathrm{i}\left(\omega_{0}-\omega\right)+\beta$

Eq. (5) reads

$\alpha E=E_{0} / 2 \pi$

which is equivalent to Eq. (7a) of our paper [2]. Eq. (6) (multiplyed with the concentration $c$ of the oscillators) is the equivalent of Eq. (7a) in [2].

To calculate the line shape for an oscillator exchanging between two positions $A$ and $B$ with frequencies $\omega_{A}$ and $\omega_{B}$ and with average lifetimes $\tau_{\mathrm{A}}$ and $\tau_{\mathrm{B}}$ we write

$\alpha_{\mathrm{A}} E_{\mathrm{A}}=E_{0} / 2 \pi+E_{\mathrm{B}} / \tau_{\mathrm{B}}-E_{\mathrm{A}} / \tau_{\mathrm{A}}$

and the corresponding Eq. (8b) with interchanged indices $A$ and B. We solve Eqs. (8) for $E_{\mathrm{A}}$ and $E_{\mathrm{B}}$ and insert into Eq. (9)

$I(\omega)=c_{\mathrm{A}} \beta_{\mathrm{A}} E_{\mathrm{A}} E_{\mathrm{A}}^{*}+c_{\mathrm{B}} \beta_{\mathrm{B}} E_{\mathrm{B}} E_{\mathrm{B}}^{*}$

which is Eq. (6) for the case of exchanging oscillators $A$ and $\mathrm{B}$ with concentrations $c_{\mathrm{A}}$ and $c_{\mathrm{B}}$. Eq. (9) describes the line broadening of the spectrum due to rapid exchange. It predicts a decrease of the integrated intensity $I_{0}$ due to exchange processes as described in our earlier paper [2].

The parameter $\beta$ is equivalent to the reciprocal of the spin-spin relaxation time $T_{2}$ in NMR spectroscopy. It depends on all mechanisms which lead to a depopulation of the excited state except chemical exchange. $\beta$ can be experimentally determined provided that conditions can be chosen such that no chemical exchange is involved. In our work the constant $\beta$ for the free nitrate ion was obtained from Raman spectra of $\mathrm{NaNO}_{3}$ solutions.

The form of Eq. (9) (or rather its equivalent Eq. (13) in [2]) including the factors $\beta_{\mathrm{A}}$ and $\beta_{\mathrm{B}}$ has been derived quantum mechanically by Heitler [7]. It has been made plausible in our paper [2]. It must be realized that by omitting the factors $\beta_{\mathrm{A}}$ and $\beta_{\mathrm{B}}$ in Eq. (9), the kinetic depression effect on the spectral amplitudes is not removed.

An important issue has been discussed by Strauss and MacPhail [4] regarding the lower time range of reaction times accessible to line broadening techniques. The model of an instantaneous switching from state $A$ to $B$ and vice versa in a reaction $A \rightleftharpoons B$ is only an approximation. A finite 
transition time $\tau_{t}$ is necessary for the proper reaction, during which all parameters such as vibration frequencies, damping constants $\beta$, polarizabilities (or dipole moments) change continuously from the values valid in state $A$ to those valid in state $\mathbf{B}$. This time will be of the order of a vibration period. Therefore, the inequality (10) must hold

$v_{\mathrm{i}} \tau_{\mathrm{i}} \gg 1 \quad \mathrm{i}=\mathrm{A}$ or $\mathrm{B}$

Whereas in NMR spectroscopy Eq. (10) is excellently fulfilled, this may not be the case with exchange broadened bands in vibrational spectroscopy. With typical values in our measurements on nitric acid $\tau=10^{-12} \mathrm{~s}$ and a Raman wave number of $1000 \mathrm{~cm}^{-1}$ the product $v \tau$ is 30 . Therefore, the assumption that reaction rate and $\beta$-relaxation are independent of each other, will not be too bad an approximation. However, chemical reaction times shorter than about $10^{-13} \mathrm{~s}$ are ill defined physical quantities.

Three approaches to the problem of chemical exchange and line broadening of vibrational spectra exist:

1) The formula of Kreevoy and Mead [6] which is claimed to be valid for the "slow case" $(\Delta \omega \cdot \tau \gg 1)$. It is quite analogue to the Bloch equations of NMR.

2) The formula of Strauss et al. [1,3] and

3) The approach of Strehlow et al. $[2,8]$.

Fortunately, the line shapes calculated are very similar with all three methods. Therefore older results which have been evaluated using the Kreevoy-Mead technique will not be obsolete. The important difference in the three formulas is the dependence of the integrated intensity of the spectrum on the kinetics. Method 1) and 2) predict only a small dependence whereas according to 3) strong kinetic amplitude damping effects may occur. What is the experimental evidence?

\section{Experiments}

Unfortunately, most of the earlier measurements of kinetically broadened vibrational spectra have only been discussed in terms of line shape, neglecting consideration of total intensity. One of the few exceptions is the measurement of the interconversion of rotamers of 1,2-dichloroethane at different temperatures by Cohen and Weiss [9]. Two series of infrared spectra, one in the neat liquid and one in at $4 \% \mathrm{v} / \mathrm{v}$ solution in $\mathrm{Cs}_{2}$, are displayed in Fig. 1. The effect of the kinetics on the total intensity is clearly visible.

These authors in a later paper [10] presented kinetically broadened IR-spectra of acetylacetone where, unfortunately, "for concenience' of presentation all specira were normalized to the same height".

The most informative relevant measurements have been performed by Irish and Puzic [11] and by [2] with nitric acid. The Raman spectra show strong bands between 900 and $1100 \mathrm{~cm}^{-1}$. The precision of the calibrated measurements is very good. It has been demonstrated in [2] that the depression of the total intensity at higher rates cannot be explaincd by assuming different Raman sensitivities of $\mathrm{HNO}_{3}$ and of $\mathrm{NO}_{3}^{-}$.

A further (not yet published), possibility to obtain rate constants for the nitric acid system from total intensity ratios alone will be presented now. As shown in [8] the ratio $F$ of the Raman total intensity of the exchanging system ( $2 \mathrm{M} \mathrm{HNO}_{3}$ ) to that of the nonexchanging system (2 $\mathrm{M} \mathrm{NaNO}_{3}$ ) is given by

$F=\beta \tau(1+\beta \tau)$
Eq. (11) holds strictly only for the slow case with a reaction of the type $\mathrm{A} \rightleftharpoons \mathrm{B}$. In nitric acid we have three species of nitrate: free $\mathrm{NO}_{3}^{-}$, a solvent separated ion pair $\mathrm{NO}_{3}^{-} \mathrm{H}_{2} \mathrm{OH}^{+}$and undissociated nitric acid $\mathrm{HNO}_{3}$. However, in $2 \mathrm{M}$ nitric acid the two latter species are at low concentrations and are not visible in the Raman spectrum. Thus we may apply Eq. (11) to the band of the free nitrate ion. $\beta=3.9 \mathrm{~cm}^{-1}=7.35 \cdot 10^{11} \mathrm{rd} \mathrm{s}^{-1}$ was obtained from a $\mathrm{NaNO}_{3}$ solution. The ratio of the total intensity $F$ for $2 \mathrm{M} \mathrm{HNO}_{3}$ and $2 \mathrm{M}$ $\mathrm{NaNO}_{3}$ was experimentally found to be 0.89 . From these experimentally determined values we calculate an average lifetime for the nitrate ion of $11.0 \mathrm{ps}$ in $2 \mathrm{M}$ nitric acid which compares favourably with the value $11.8 \mathrm{ps}$ calculated with the full fitting program. It should be realized that these two data are independent of each other. Their equality lends further support to the theory that fast kinetics is the reason for the observed amplitude depression.
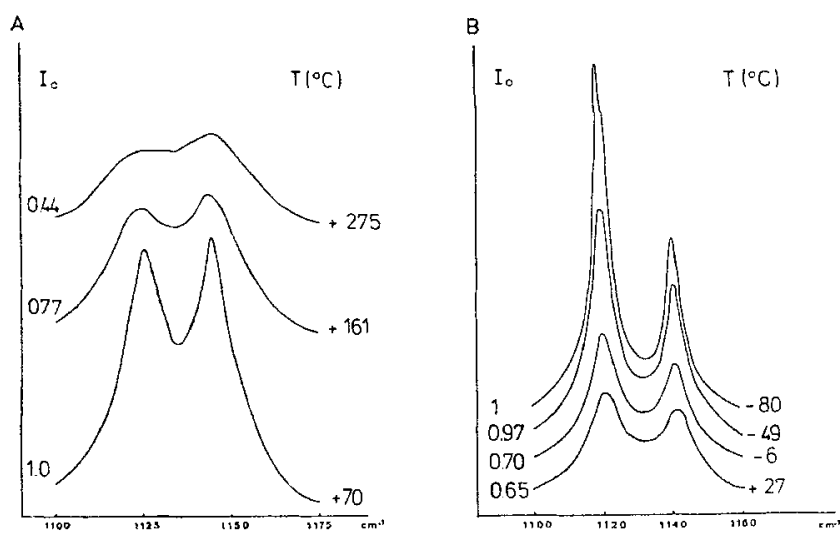

Fig. 1

A Spectra of liquid 1,2-dichloroethane at different temperatures [9].

B Spectra of 1,2-dichloroethane in $\mathrm{CS}_{2}(4 \% \mathrm{v} / \mathrm{v})$ at different temperatures [9].

The relative integrated intensities $I_{0}$ are precise to only about $\pm 5 \%$ due to uncertainties in the base lines.

Adapted with permission from [9]. Copyright 1983 American Chemical Society

Another argument in favor of this interpretation is the effect of $\mathrm{HCl}$ on $F$ for the nitrate ion in $2 \mathrm{M}$ nitric acid. For the concentrations of $\mathrm{HCl} 0,4$, and $5.82 \mathrm{M}$ the ratio $F$ was found to be 0.89 , 0.75 , and 0.62 . The lowest value of $F(=0.47)$ was observed in $14.7 \mathrm{M}$ $\mathrm{HNO}_{3}$.

\section{References}

[1] K. H. Wood and H. L. Strauss, Ber. Bunsenges. Phys. Chem. 93, $615(1989)$.

[2] H. Strehlow, I. Wagner, and P. Hildebrandt, Ber. Bunsenges. Phys. Chem. 87, 516 (1983).

[3] R. A. MacPhail and H. L. Strauss, J. Chem. Phys. 82, 1156 (1985).

[4] W. Heitler, The Quantum Theory of Radiation Third Ed. \$4, Clarendon Press, Oxford 1954

[5] Eq. (28) of [4].

[6] M. M. Kreevoy and C. A. Mead, J. Am. Chem. Soc. 84, 4596 (1962).

[7] W. Heitler, [4], §18

[8] H. Strehlow, Ber. Bunsenges. Phys. Chem. 92, 467 (1988)

[9] B. Cohen and S. Weiss, J. Phys. Chem. 87, 3606 (1983).

[10] B. Cohen and S. Weiss, J. Phys. Chem. 88, 3159 (1984)

[11] D. E. Irish and O. Puzic, J. Solution Chem. 10, 377 (1981).

(Eingegangen am 16. Januar 1989, E 7003 endgültige Fassung am 16. Februar 1989) 